$\mathrm{m} 46.005$

\title{
Strain Engineering of Multiferroic Thin Films
}

Christophe J.M. Daumont $^{1}$, B., Peter Fonteijn ${ }^{1}$, B. Noheda1 and Gustau Catalan ${ }^{1,2}$.

${ }^{1}$ Solid State Chemistry Laboratory, Materials Science Centre, University of Groningen, Nijenborgh 4, 9747 AG Groningen, The Netherlands.

${ }^{2}$ Department of Earth Sciences, University of Cambridge, Downing Street, Cambridge CB2 3EQ, United Kingdom.

\section{Keywords: Multiferroic, Strain engineering, Synchrotron Radiation}

Multiferroics are materials exhibiting ferroelectricity and magnetism simultaneously. From the applications point of view they are also very interesting since they present the possibility to manipulate their electric and magnetic moments by magnetic and electric fields, respectively, if the two order parameters are coupled. While efforts have been devoted to search and design new multiferroics, the nature of the coupling between ferroelectricity and magnetism is still under investigation and it greatly differs from system to system[1]. Using epitaxial growth, the properties of multiferroic thin films can be engineered by tuning the strain imposed by the substrate. Among the known multiferroics, the perovskite oxides, such as $\mathrm{BiFeO}_{3}$ or $\mathrm{TbMnO}_{3}$, are very interesting candidates for epitaxial growth because of their small lattice mismatch with, for instance, $\mathrm{SrTiO}_{3}$ substrates. Using the Pulsed Laser Deposition technique (PLD), epitaxially grown $\mathrm{BiFeO}_{3}$ and $\mathrm{TbMnO}_{3}$ thin films on $\mathrm{SrTiO}_{3}$ substrates have been reported with thicknesses of tens of nanometers [2]. Recently, ultrathin films of $\mathrm{BiFeO}_{3}$ have been also reported down to $5 \mathrm{~nm}$ [3], but the characterization of such thin layers by means of standard X-ray diffraction is not easy because of the limited diffracted intensity. As far as we know, there are no reports of neither fully-strained $\mathrm{TbMnO}_{3}$ films nor of $\mathrm{TbMnO}_{3}$ films below $100 \mathrm{~nm}$. Growth and characterization of ultrathin films is not only of fundamental importance, but it is also significant in the context of multilayer growth, in order to gain control of the behavior and thickness limitations of the individual layers, which are usually very thin. Here we report the successful growth of epitaxial ultrathin $(5 \mathrm{~nm}) \mathrm{BiFeO}_{3}$ and $\mathrm{TbMnO}_{3}$ films on $\mathrm{SrTiO}_{3}$ single crystals via RHEED-assisted PLD and their structural characterization using synchrotron diffraction in grazing-incidence geometry, along with highresolution laboratory X-ray diffraction. Atomic Force Microscopy was used to investigate the surface of the films. Thickness dependence and the strain relaxation of the grown films is also reported. We show that for thicknesses below $\sim 50 \mathrm{~nm}, \mathrm{BiFeO}_{3}$ thin films are tetragonal and that above that thickness they start to relax via a monoclinic distortion, still different from the fully relaxed (bulk) rhombohedral phase.

[1] Nicola A. Spaldin and Manfred Fiebig, Science 309, 5733 (2005), N. M. Fiebig, J. Phys.D, R123 (2005); T. Kimura et al., Nature 426, 55 (2003) and N. Hur et al.,Nature 429, 392 (2004)

[2] J.Wang, et al., Science 299, 1719 (2003) ; W. Eerenstein et al. Science 307, 1203 (2005) ; Y. M. Cui et al., APL 86, 203501 (2005) ; G. Xu et al., APL 86, 182905 (2005) ; X. Qi et al., APL 86, 071913 (2005).

[3] H. Béa et al., APL 88, 062502 (2006) 